PROCEEDINGS OF THE

AMERICAN MATHEMATICAL SOCIETY

Volume 140, Number 12, December 2012, Pages 4063-4074

S 0002-9939(2012)11271-7

Article electronically published on April 5, 2012

\title{
RAYNAUD-MUKAI CONSTRUCTION AND CALABI-YAU THREEFOLDS IN POSITIVE CHARACTERISTIC
}

\author{
YUKIHIDE TAKAYAMA
}

(Communicated by Lev Borisov)

\begin{abstract}
In this article, we study the possibility of producing a CalabiYau threefold in positive characteristic which is a counterexample to Kodaira vanishing. The only known method to construct the counterexample is the so-called inductive method such as the Raynaud-Mukai construction or Russel construction. We consider Mukai's method and its modification. Finally, as an application of the Shepherd-Barron vanishing theorem of Fano threefolds, we compute $H^{1}\left(X, H^{-1}\right)$ for any ample line bundle $H$ on a Calabi-Yau threefold $X$ on which Kodaira vanishing fails.
\end{abstract}

\section{INTRODUCTION}

Although every K3 surface in positive characteristic can be lifted to characteristic 0 2, there are some non-liftable Calabi-Yau threefolds, namely a smooth threefold $X$ with trivial canonical bundle and $H^{1}\left(X, \mathcal{O}_{X}\right)=H^{2}\left(X, \mathcal{O}_{X}\right)=0$. If a Calabi-Yau polarized threefold $(X, L)$ over the field $k$ of $\operatorname{char}(k)=p \geq 3$ is a counterexample to Kodaira vanishing, i.e., $H^{i}\left(X, L^{-1}\right) \neq 0$ for $i=1$ or $i=2, X$ is non-liftable to the second Witt vector ring $W_{2}(K)$ (and the Witt vector ring $W(k)$ ) by the celebrated Raynaud-Deligne-Illusie version of the Kodaira vanishing theorem [3. But this does not necessarily imply that $X$ cannot be liftable to characteristic 0 . Moreover, a nonliftable variety is not necessarily a counterexample to Kodaira vanishing and as far as the author is aware, it is not known whether Kodaira vanishing holds for the non-liftable Calabi-Yau threefolds [6, 7, 8, 16, 4, 1, that have been found so far. We do not even know whether Kodaira vanishing holds for all Calabi-Yau threefolds. Thus the Kodaira type vanishing for Calabi-Yau threefolds is an interesting problem which is independent from but seems to be closely related to the lifting problem.

A counterexample to Kodaira vanishing which is a surface over a curve [14 has been given by M. Raynaud. This example was extended to arbitrary dimension by S. Mukai [11, 12, which we will call the Raynaud-Mukai construction or, simply, the Mukai construction.

The idea is, so to say, an inductive construction. Namely, we start from a polarized smooth curve $(C, D)$. The ample divisor $D$ satisfies a special condition, which is a sufficient condition for the non-vanishing $H^{1}\left(X, \mathcal{O}_{X}(-D)\right) \neq 0$, and called a (pre-)Tango structure. Then we give an algorithm to construct from a variety $X$ with a (pre-)Tango structure $D$ a new variety $\tilde{X}$ with a higher dimensional (pre-)Tango structure $\tilde{D}$ such that $\operatorname{dim} \tilde{X}=\operatorname{dim} X+1$, using the cyclic cover

Received by the editors October 18, 2010 and, in revised form, May 2, 2011 and May 21, 2011. 2010 Mathematics Subject Classification. Primary 14F17, 14J32; Secondary 14M99, 14J45.

(C)2012 American Mathematical Society 4063

Reverts to public domain 28 years from publication 
technique. There is another way of constructing counterexamples using quotients of $p$-closed differential forms [15, 19]. But this is also an inductive construction, and the obtained varieties are the same as the Raynaud-Mukai construction [19]. As far as the author is aware, a non-inductive construction of higher dimensional counter-examples is not yet found.

In this paper, we consider the problem of whether we can construct a Calabi-Yau threefold with Kodaira non-vanishing by Mukai construction or by its modification. Section 2 presents the Raynaud-Mukai construction. For $p \geq 5$, Raynaud-Mukai varieties are of general type so that the only possibility resides in the cases of $p=2,3$. Then in section 3 , we will see that Mukai construction does not produce any K3 surfaces or Calabi-Yau threefolds (Corollary 3.2 and Corollary 3.3). Then we consider possible modifications of the Raynaud-Mukai construction: we keep the inductive construction but give up obtaining a (pre-)Tango structure. We show that if there exists a surface $X$ of general type together with a (pre-)Tango structure $D$ satisfying some property (this is not obtained by Mukai construction), we can construct a Calabi-Yau threefold $\tilde{X}$ with a (pre-)Tango structure $\tilde{D}$ (Corollary 3.4) and describe the cohomology $H^{1}\left(\tilde{X}, \mathcal{O}_{\tilde{X}}\right)$ in certain situations (Proposition [3.6). Unfortunately, we could not prove or disprove existence of such a polarized surface $(X, D)$.

Finally, in section 3 we show that if Kodaira non-vanishing $H^{1}\left(X, L^{-1}\right) \neq 0$ holds for a polarized Calabi-Yau threefold $(X, L)$ over the field $k$ of char $k=p \geq 5$ satisfying the condition that $L^{\ell}$ is a Tango-structure for some $\ell \geq 1$, we compute the cohomology $H^{1}\left(X, H^{-1}\right)$ for any ample line bundle $H$ of $X$ (Theorem 4.2. Corollary 4.3).

\section{The Raynaud-Mukai CONSTRuction}

In this section, we present the Raynaud-Mukai construction. Although 12 is available now, we prefer to use the version described in [11, which is slightly different from the 2005 version. As the 1979 version is only available in Japanese, we present some details for the reader's convenience.

The idea is to construct from a counterexample to Kodaira vanishing, i.e., a polarized variety $(X, L)$ with $H^{1}\left(X, L^{-1}\right) \neq 0$, a new counterexample $(\tilde{X}, \tilde{L})$ with $\operatorname{dim} \tilde{X}=\operatorname{dim} X+1$. This inductive construction starts from a polarized curve $(X, L)$ called a Tango-Raynaud curve.

\subsection{Pre-Tango structure and Kodaira non-vanishing.}

Definition 2.1 (pre-Tango structure). Let $X$ be a smooth projective variety. Then an ample divisor $D$, or an ample line bundle $L=\mathcal{O}_{X}(D)$, is called a pre-Tango structure if there exists an element $\eta \in k(X) \backslash k(X)^{p}$, where $k(X)$ denotes the function field of $X$, such that the Kähler differential is $d \eta \in \Omega_{X}(-p D)$, which will be simply denoted as $(d \eta) \geq p D$. In this paper, the element $\eta$ will be called a justification of the pre-Tango structure.

Existence of a pre-Tango structure implies Kodaira non-vanishing. In fact, consider the absolute Frobenius morphism

$$
F: \mathcal{O}_{X}(-D) \longrightarrow \mathcal{O}_{X}(-p D)
$$

such that $F(a)=a^{p}$ for $a \in \mathcal{O}_{X}$ and set $B_{X}(-D):=\operatorname{Coker} F$. Then we have

$$
0 \longrightarrow H^{0}\left(X, B_{X}(-D)\right) \longrightarrow H^{1}\left(X, \mathcal{O}_{X}(-D)\right) \stackrel{F}{\longrightarrow} H^{1}\left(X, \mathcal{O}_{X}(-p D)\right),
$$


and then we can show

Proposition 2.2. $H^{0}\left(X, B_{X}(-D)\right)=\{d f \in k(X) \mid(d f) \geq p D\}$.

Thus, if there exists a pre-Tango structure $D$ and $\operatorname{dim} X \geq 2$, then we have Kodaira non-vanishing: $H^{1}\left(X, \mathcal{O}_{X}(-D)\right) \neq 0$.

Notice that the inclusion $H^{0}\left(X, B_{X}(-D)\right) \subset H^{1}\left(X, \mathcal{O}_{X}(-D)\right)$ may be strict, so that there is a possibility that a non-pre-Tango structure $L$ causes a Kodaira non-vanishing. However, since the iterated Frobenius map

$$
F^{e}: H^{1}\left(X, L^{-1}\right) \longrightarrow H^{1}\left(X, L^{-p^{e}}\right)
$$

is trivial for $e \gg 0, L^{n}$ is a pre-Tango structure for sufficiently large $n \in \mathbb{N}$.

The pre-Tango structure for curves is characterized by the Tango-invariant [21, 20 . Let $C$ be a smooth projective curve of genus $g(\geq 2)$. Then the Tango-invariant is defined as

$$
n(C)=\max \left\{\operatorname{deg}\left[\frac{d f}{p}\right]: f \in k(X) / k(X)^{p}\right\},
$$

where $[\cdots]$ denotes the round up. We easily know that

$$
0 \leq n(C) \leq \frac{2(g-1)}{p} .
$$

Then, $C$ has a pre-Tango structure $D$ if $n(C)>0$. We just set $D=\left[\frac{(d f)}{p}\right]$ and then $D$ is ample on $C$ such that $(d f) \geq p D$.

In the following, we will call the pair $(X, L)$ in Definition 2.1 a pre-Tango polarization. The Raynaud-Mukai construction is an algorithm to make a new pre-Tango polarization from a pre-Tango polarization whose dimension is lower by one.

2.2. Purely inseparable cover. From a pre-Tango polarized variety $(X, L)$ we can construct a reduced and irreducible purely inseparable cover $\tau: G \longrightarrow X$ of degree $p$. Conversely, the existence of such a cover implies the existence of a pre-Tango polarization.

2.2.1. Construction and characterization. Given a pre-Tango polarized variety $(X$, $\left.L=\mathcal{O}_{X}(D)\right)$, choose an element $(0 \neq) \eta \in H^{0}\left(X, B_{X}(-D)\right)(=\operatorname{Ker} F)$. Then we have a corresponding non-split short exact sequence

$$
0 \longrightarrow \mathcal{O}_{X} \longrightarrow E \longrightarrow L \longrightarrow 0,
$$

where $E$ is a rank 2 vector bundle on $X$. Taking the Frobenius pull-back, we obtain an exact sequence

$$
0 \longrightarrow \mathcal{O}_{X} \longrightarrow E^{(p)} \longrightarrow L^{(p)} \longrightarrow 0
$$

where, for example, $E^{(p)}=E \otimes_{\mathcal{O}_{X}} \mathcal{O}_{X^{\prime}}$ with $F: \mathcal{O}_{X} \longrightarrow \mathcal{O}_{X^{\prime}}$ the Frobenius morphism. Notice that the new sequence corresponds to $F(\eta)=0$ so that it splits and by using the split maps, we obtain the sequence with the reverse arrows

$$
0 \longleftarrow \mathcal{O}_{X} \longleftarrow E^{(p)} \longleftarrow L^{(p)} \longleftarrow 0 .
$$

Tensoring by $L^{(p)^{-1}}$ over $\mathcal{O}_{X}$, we finally obtain the sequence

$$
0 \longrightarrow \mathcal{O}_{X} \longrightarrow E^{(p)} \otimes L^{(p)^{-1}} \longrightarrow L^{(p)^{-1}} \longrightarrow 0 .
$$

Now we consider the $\mathbb{P}^{1}$-fibration

$$
\pi: P=\mathbb{P}(E) \longrightarrow X
$$


together with the canonical section $F \subset P$, which is defined by the image of $1 \in \mathcal{O}_{X}$ in $E$, and

$$
\pi^{(p)}: P^{(p)}=\mathbb{P}\left(E^{(p)} \otimes L^{(p)^{-1}}\right) \cong \mathbb{P}\left(E^{(p)}\right) \longrightarrow X
$$

together with the canonical section $F^{(p)} \subset P^{(p)}$ which is the image of $1 \in \mathcal{O}_{X}$ in $E^{(p)} \otimes L^{(p)^{-1}}$, corresponding to (2.1) and (2.2). Moreover, we consider the relative Frobenius morphism $\psi: P \longrightarrow P^{(p)}$ over $X$. On an open set $U \subset X$ such that $\left.E\right|_{U} \cong \mathcal{O}_{U}^{r}$ with $r=\operatorname{rank} E, \psi$ is induced by the local morphism $\left.E^{(p)}\right|_{U} \cong$ $\left.\mathcal{O}_{U}^{r} \otimes_{\mathcal{O}_{U}} \mathcal{O}_{U^{\prime}} \rightarrow E\right|_{U}$ sending $\sum_{i=1}^{r} a_{i} \otimes f=\sum_{i} 1 \otimes a_{i}^{p} f \in \mathcal{O}_{U}^{r}$ to $\sum_{i} a_{i}^{p} f \in \mathcal{O}_{U}^{r}$. Thus, on a fiber $\pi^{-1}(x) \cong \mathbb{P}^{1},\left.\psi\right|_{\pi^{-1}(x)}: \pi^{-1}(x) \longrightarrow \pi^{(p)^{-1}}(x)$ is the Frobenius pull-back, i.e., $\psi(a, b)=\left(a^{p}, b^{p}\right)$ for every projective coordinate $(a, b) \in \pi^{-1}(x)$. Now consider the scheme-theoretic inverse image of $F^{(p)}$ inside $P$ :

$$
G:=\psi^{-1}\left(F^{(p)}\right) \subset P .
$$

Then we can show

Proposition 2.3. $\quad$ (1) $G \cap F=\emptyset$,

(2) $\mathcal{O}_{P}(G) \cong \mathcal{O}_{P}(p) \otimes \pi^{*} L^{-p} \cong \mathcal{O}_{P}\left(p F-p \pi^{*} D\right)$, and

(3) $\rho=\left.\pi\right|_{G}: G \longrightarrow X$ is a purely inseparable cover of degree $p$.

We can show that the existence of such a $G$ characterizes the pre-Tango structure. To summarize, we have

Theorem 2.4 (See Proposition 1.1 in [12]). Let $X$ be a smooth projective variety of characteristic $p>0$ and $L$ be an ample line bundle. Then the following are equivalent:

(1) $L$ is a pre-Tango structure.

(2) There exists a $\mathbb{P}^{1}$-bundle $\pi: P \longrightarrow X$ and a reduced irreducible effective divisor $G \subset P$ such that

(a) $\rho: G \longrightarrow X$ is a purely inseparable cover of degree $p$;

(b) $P=\mathbb{P}(E)$, where $E$ is a rank 2 vector bundle on $X$ such that

$$
0 \longrightarrow \mathcal{O}_{X} \longrightarrow E \longrightarrow L \longrightarrow 0 \text {. }
$$

2.2.2. Smoothness. For smoothness of the purely inseparable cover $G$, we have

Theorem 2.5 (S. Mukai [12]). Let $(X, D)$ be a pre-Tango polarized variety over the field of characteristic $p>0$ and let $G$ be the purely inseparable cover constructed from a justification $(0 \neq) \eta \in k(X) \backslash k(X)^{p}$. Then $G$ is smooth if and only if $(d \eta)=$ $p D$. This means that for the multiplication by $d \eta$,

$$
\mathcal{O}_{X}(p D) \stackrel{d \eta}{\longrightarrow} \Omega_{X} \longrightarrow \operatorname{Coker}(d \eta) .
$$

Coker $d \eta$ is locally free at every $x \in X$.

Proof. For a proof in the case of $\operatorname{dim} X=2$, see Theorem 3 [18].

Definition 2.6 (Tango structure). Let $X$ be a smooth projective variety with a pre-Tango structure $L=\mathcal{O}_{X}(D)$. Then $D$, or $L$, is called a Tango structure if and only if a justification $\eta \in k(X) \backslash k(X)^{p}$ satisfies $(d \eta)=p D$. In this case, the pre-Tango polarization $(X, L)$ or $(X, D)$ will be called a Tango polarization.

A smooth projective curve $X$ of genus $g \geq 2$ with a Tango structure $D$ is called a Tango-Raynaud curve. For examples of Tango-Raynaud curves, see for example [14, 11, 12. 
2.3. Cyclic cover. Let $(X, D)$ be a pre-Tango polarization, where $D$ is divided by $k \in \mathbb{N}$ with $(p, k)=1$ and we have $D=k D^{\prime}$. If $X$ is a curve, we can divide $D$ by any natural number $k$ dividing $\operatorname{deg} D$ using the theory of Jacobian varieties (cf. page 62 of [13]). But the condition $(p, k)=1$ is necessary for the covering to be cyclic.

Now we construct a $k$ th cyclic cover of the $\mathbb{P}^{1}$-fibration $\pi: P \longrightarrow X$ ramified over $F+G$, which means that $\pi$ is ramified at the reduced preimage of $F+G$. There are at least two well-known constructions.

The first one is rather explicit and is suitable for computing cohomologies (cf. [18]). We first choose $m \in \mathbb{N}$ such that $k \mid(p+m)$ and set $\mathcal{M}=\mathcal{O}_{P}\left(-\frac{p+m}{k} F\right) \otimes$ $\pi^{*} \mathcal{O}_{X}\left(p D^{\prime}\right)$. Then we have $\mathcal{M}^{\otimes k}=\mathcal{O}_{P}(-m F) \otimes \mathcal{O}_{P}(-p F) \otimes \pi^{*} \mathcal{O}_{X}(p D)=$ $\mathcal{O}_{P}(-m F-G)$ by Proposition 2.3. Then we can introduce $\bigoplus_{i=0}^{k-1} \mathcal{M}^{\otimes i}$ the structure of a graded $\mathcal{O}_{P}$-algebra by defining multiplication $\mathcal{M}^{\otimes i} \times \mathcal{M}^{\otimes j} \longrightarrow \mathcal{M}^{\otimes i+j}$ s.t. $(a . b) \mapsto a \otimes b$ if $i+j<k$ and $\mathcal{M}^{\otimes i} \times \mathcal{M}^{\otimes j} \longrightarrow \mathcal{M}^{\otimes i+j} \longrightarrow \mathcal{M}^{\otimes i+j-k}$ s.t. $(a . b) \mapsto a \otimes b \mapsto a \otimes b \otimes \xi$ if $i+j \geq k$, where we choose a non-trivial element $\xi \in \mathcal{O}_{P}(m F+G)$ such that $m F+G$ is the zero locus of $\xi$. Now we consider the affine morphism $X^{\prime}:=\operatorname{Spec} \bigoplus_{i=0}^{k-1} \mathcal{M}^{\otimes i} \rightarrow P$, and this is the cyclic cover ramified over $m F+G$. Since $X$ is smooth, $F \cong X$ is also smooth. Moreover if $D$ is a Tango structure and $G$ is smooth by Theorem 2.5, then $X^{\prime}$ is smooth if and only if $m=1$; if $m>1$, then $X^{\prime}$ is singular along $F$, which may cause non-normality of $X^{\prime}$. Normalization of $X^{\prime}$, if necessary, is carried out by the Esnault-Viehweg method (see $\S 3$ of [5] ). $\tilde{X}=\mathcal{S} p e c \bigoplus_{i=0}^{k-1} M^{\otimes i} \otimes \mathcal{O}_{P}\left(\left[\frac{i(m F+G)}{k}\right]\right)$, and this is smooth if $D$ is Tango. We note that this normalization procedure highly depends on the condition $(p, k)=1$ since we use the $k$ th root of unity. Then we set the natural morphism $\varphi: \tilde{X} \longrightarrow X^{\prime} \longrightarrow P$.

The second construction uses normalization. Since we have the linear equivalence $G \sim p F-p \pi^{*}(D)$ there exists a function $R \in k(P)$ such that $(R)=G-(p F-$ $\left.p \pi^{*}(D)\right)=G-\left(p F-p k \pi^{*}\left(D^{\prime}\right)\right)$. Then let $\tilde{X}$ be the normalization of $P$ in the finite extension $k(P)\left(R^{1 / k}\right)$ of $k(P)$ and let $\varphi: \tilde{X} \longrightarrow P$ be the normalization morphism. Then we set $f=\pi \circ \varphi$. Now if we work locally we know that there exist divisors $\tilde{G}$ and $\tilde{F}$ on $\tilde{X}$ such that $\varphi^{*} F=k \tilde{F}$ and $\varphi^{*} G=k \tilde{G}$. Moreover, we have $\tilde{G} \sim p \tilde{F}-p f^{*}\left(D^{\prime}\right)$ on $\tilde{X}$. We note that the condition $(p, k)=1$ is necessary to assure the existence of $\tilde{F}$, division of $F$ by $k$. Otherwise, if $k=p^{\ell} r$ with $\ell \geq 1$ and $(p, r)=1$ we have $\tilde{F} \subset \tilde{X}$ such that $\varphi^{*} F=k^{\prime} \tilde{F}$ with $k^{\prime}=p^{\ell-1} r=k / p$. $\tilde{X}$ is smooth if $D$ is Tango.

Now we set $f:=\pi \circ \varphi: \tilde{X} \longrightarrow X$, which is actually a fibration of rational curves with moving singularities, i.e., rational curves with cusp singularity of type $x^{p}=y^{t}$ at $\tilde{G}$.

2.4. Polarization. The cyclic cover $\tilde{X}$ of the $\mathbb{P}^{1}$-fibration is a counterexample to Kodaira vanishing because the polarization $\tilde{D}=(k-1) \tilde{F}+f^{*}\left(D^{\prime}\right)$ causes nonvanishing $H^{1}\left(\tilde{X}, \mathcal{O}_{\tilde{X}}(-\tilde{D})\right) \neq 0$. In fact, $\tilde{D}$ is ample (see Sublemma $1.6[12]$ ) and we have

Proposition 2.7. Suppose $\tilde{X}$ is as above. Then $\tilde{D}$ is a Tango structure of $\tilde{X}$ and in particular we have Kodaira non-vanishing $H^{1}\left(\tilde{X}, \mathcal{O}_{\tilde{X}}(-\tilde{D})\right) \neq 0$. 
This result is stated in [11] without proof and in the case of $k \equiv 1 \bmod p$ a proof using Maruyama's elementary transformation [10] is given in [12]. We give here a proof of the general case.

Proof. Let $\tilde{\eta}=R^{1 / k} \in k(\tilde{X})$. Since $(\tilde{\eta})=\tilde{G}-p \tilde{F}+p f^{*}\left(D^{\prime}\right), \tilde{\eta}$ is locally described as $\tilde{\eta}=g\left(\delta \phi^{-1}\right)^{p}$, where $g, \phi$ and $\delta$ are local equations defining $\tilde{G}, \tilde{F}$ and $f^{*}\left(D^{\prime}\right)$. Then its Kähler differential is

$$
d \tilde{\eta}=\left(\delta \phi^{-1}\right)^{p} d g=\left(\phi^{k-1} \delta\right)^{p} \phi^{-p k} d g .
$$

Now we consider $d g$. As a Cartier divisor we describe $D=\left\{\left(U_{i}, g_{i}\right)\right\}_{i}$ for an open cover $X=\bigcup_{i} U_{i}$ and $g_{i} \in k(X)$. Since $D$ is a pre-Tango structure, there exists a justification $\eta \in k(X)$ such that $(d \eta) \geq p D$, which locally means that we have $\left.\eta\right|_{U_{i}}=g_{i}^{p} c_{i}$ for some $c_{i} \in \mathcal{O}_{U_{i}}$ so that we have $\left.(d \eta)\right|_{U_{i}}=g_{i}^{p} d c_{i}$. Then, as in Proposition 1 [18], $G \subset P$ is locally described as

$$
\operatorname{Proj} \mathcal{O}_{U_{i}}[x, y] /\left(c_{i} x^{p}+y^{p}\right),
$$

where $x$ is the (local) coordinate corresponding to the canonical section $F$ of $\pi$ : $P \longrightarrow X$. Hence the local defining equation of $G \subset P$ is $c_{i} x^{p}+y^{p}$, and since $\varphi^{*} F=k \tilde{F}$ and $\varphi^{*} G=k \tilde{G}$, the defining equation of $\tilde{G}$ is $g=c_{i} Z^{k p}+W^{k p}$, where $Z$ is the local coordinate of $\tilde{X}$ corresponding to $\tilde{F}$, namely $Z=\epsilon \phi$ with some local unit $\epsilon$. Thus we have

$$
d g=\epsilon^{p k} \phi^{p k} d c_{i} .
$$

Thus by (2.3) and (2.4) we obtain $d \tilde{\eta}=\left(\delta \phi^{-1}\right)^{p} d g=\epsilon^{p k}\left(\phi^{k-1} \delta\right)^{p} d c_{i}$ so that

$$
(d \tilde{\eta}) \geq p\left((k-1) \tilde{F}+f^{*} D^{\prime}\right)=p \tilde{D},
$$

where the equality holds if $(d \eta)=p D$, i.e., if $D$ is a Tango structure.

\section{Calabi-Yau threefolds and the Raynaud-Mukai construction}

3.1. Raynaud-Mukai varieties cannot be Calabi-Yau. The aim of this section is to show that the Mukai construction does not produce K3 surfaces or Calabi-Yau threefolds. Notice that the Raynaud-Mukai variety is always of general type for $p \geq 5$ (cf. Prop. 7 [11] or Prop. 2.6 [12]) so that the only possibility is the case $p=2,3$.

Now let $(X, D), D=k D^{\prime}(k \in \mathbb{N}), \pi: P \longrightarrow X, F, G \subset P,(\tilde{X}, \tilde{D}), \varphi: \tilde{X} \longrightarrow P$ and $f: \tilde{X} \longrightarrow X$ be as in the previous section. The canonical divisor of $X$ will be simply denoted by $K$. Now we have

Proposition 3.1 (cf. Prop. 7 [1]). Let $\tilde{K}$ be the canonical divisor of $\tilde{X}$. Then we have

$$
\tilde{K} \sim(p k-p-k-1) \tilde{F}+f^{*}\left(K-(p k-p-k) D^{\prime}\right) .
$$

Proof. Since the finite morphism $\varphi: \tilde{X} \longrightarrow P$ is ramified at $\tilde{F}=\left(\varphi^{*}(F)\right)_{\text {red }}$ and $\tilde{G}=\left(\varphi^{*}(G)\right)_{r e d}$ with the same ramification index $k$ and $F+G \sim(p+1) F-p k \pi^{*} D^{\prime}$, we compute

$$
\begin{aligned}
\tilde{K} & \sim \varphi^{*} K_{P}+(k-1)(\tilde{F}+\tilde{G}) \quad \text { by the ramification formula } \\
& \sim \varphi^{*} K_{P}+(k-1) \frac{1}{k} \varphi^{*}(F+G) \\
& \sim \varphi^{*} K_{p}+(k-1)\left((p+1) \tilde{F}-p f^{*} D^{\prime}\right) .
\end{aligned}
$$


Moreover, since $E$ is the rank 2 vector bundle satisfying

$$
0 \longrightarrow \mathcal{O}_{X} \longrightarrow E \longrightarrow \mathcal{O}_{X}\left(k D^{\prime}\right) \longrightarrow 0,
$$

we have $K_{P} \sim-2 F+\pi^{*}\left(K+k D^{\prime}\right)$. Then we obtain the required formula.

We notice that since Pic $P \cong \mathbb{Z} \cdot[F] \oplus \pi^{*} \operatorname{Pic} X$ and $\varphi$ is finite, we have Pic $\tilde{X} \cong$ $\mathbb{Z} \cdot[\tilde{F}] \oplus f^{*}$ Pic $X$. This fact will be used implicitly in the following discussion.

Corollary 3.2. A Raynaud-Mukai surface can never be a K3 surface.

Proof. Assuming $\operatorname{dim} \tilde{X}=2$, we have only to show that we never have $\tilde{K} \sim 0$. Assume that we have $\tilde{K} \sim(p k-p-k-1) \tilde{F}+f^{*}\left(K-(p k-p-k) D^{\prime}\right) \sim 0$, from which we have two relations $p k-p-k-1=0$ and $K-(p k-p-k) D^{\prime}=0$. By the first relation, we have $k=\frac{p+1}{p-1} \in \mathbb{N}$, so that we must have $p=2$ and $k=3$ or $p=3$ and $k=2$. This implies that $K=D^{\prime}$ by the second relation. However, since $(X, D)$ is a (pre-)Tango polarized curve, we have $(d \eta) \geq p D$ for some justification $\eta \in k(X)$, namely $D^{\prime}=K \geq p D=p k D^{\prime}$, which is impossible unless $p k=1$.

By a similar discussion to the proof of Corollary 3.2, we can also show

Corollary 3.3. A Raynaud-Mukai threefold can never be Calabi-Yau.

Proof. Let $\tilde{X}$ be a Mukai threefold obtained from a Mukai surface $X$ with a (pre-) Tango structure $D=k D^{\prime}$ as a $k$ th cyclic cover of the $\mathbb{P}^{1}$-fibration $P$ and assume that $\tilde{K} \sim 0$. Then as in the proof of Corollary 3.2 we have $(p, k)=(2,3)$ or $(3,2)$ and

$$
K \sim D^{\prime}
$$

Now we will consider the situation whose dimensions are all lower by one. Namely, let the surface $X$ be constructed from a (pre-) Tango polarized curve $\left(X_{1}, D_{1}\right)$ with $D_{1}=k_{1} D_{1}^{\prime}$. We have the $k_{1}$ th cyclic cover $\varphi_{1}: X \longrightarrow P_{1}$ of the $\mathbb{P}^{1}$-fibering $\pi_{1}: P_{1} \longrightarrow X_{1}$ ramified over $F_{1}+G_{1}$ and $\tilde{F}_{1}=\left(\varphi_{1}^{*}\left(F_{1}\right)\right)_{\text {red }}$ and $\tilde{G}_{1}=\left(\varphi_{1}^{*}\left(G_{1}\right)\right)_{\text {red }}$ have the same ramified index $k_{1}$. We set $f_{1}=\pi_{1} \circ \varphi_{1}$. Then by Proposition 3.1. we have

$$
K \sim\left(p k_{1}-p-k_{1}-1\right) \tilde{F}_{1}+f_{1}^{*}\left(K_{1}-\left(p k_{1}-p-k_{1}\right) D_{1}^{\prime}\right) .
$$

Since we have $\left(k D^{\prime}=\right) D=\left(k_{1}-1\right) \tilde{F}_{1}+f_{1}^{*}\left(D_{1}^{\prime}\right)$ by definition, the condition (3.1) entails

$$
\left(p k_{1}-p-k_{1}-1-\frac{k_{1}-1}{k}\right) \tilde{F}_{1}+f_{1}^{*}\left(K_{1}-\left(p k_{1}-p-k_{1}+\frac{1}{k}\right) D_{1}^{\prime}\right) \sim 0 .
$$

Then the coefficient of $\tilde{F}_{1}$ must be 0 so that we have

$$
k_{1}=\frac{k(p+1)-1}{k(p-1)-1}= \begin{cases}4 & \text { if } p=2, \\ \frac{7}{3} & \text { if } p=3 .\end{cases}
$$

But since we must have $k_{1} \in \mathbb{N}$ and $\left(k_{1}, p\right)=1$, these values of $k_{1}$ are not allowed. 
3.2. A modification of the Raynaud-Mukai construction. The RaynaudMukai construction is an algorithm to construct from a given (pre-) Tango polarization $(X, D)$ with $D=k D^{\prime}$ a new (pre-)Tango polarization $(\tilde{X}, \tilde{D})$ with $\operatorname{dim} X=\operatorname{dim} \tilde{X}-1$ by taking a $k$ th cyclic cover. We apply this procedure inductively starting from a (pre-)Tango polarized curve. We have seen in the previous subsection that the essential reason that the Raynaud-Mukai construction does not produce Calabi-Yau threefolds is that we cannot find the degree $k$ cyclic covers with $(p, k)=1$ in all inductive steps.

Now we will consider some modification of the Raynaud-Mukai construction. There are the following two possibilities:

(I) Let $(X, D)$ be a (pre-) Tango polarized surface obtained by a method other than Mukai construction. Then apply the Raynaud-Mukai construction to obtain a (pre-) Tango polarized threefold $(\tilde{X}, \tilde{D})$.

(II) Let $(X, D)$ be a (pre-)Tango polarized surface by the Raynaud-Mukai construction. Then we construct a Calabi-Yau threefold in a similar way to the Mukai construction. Namely, we do not assume the condition $(p, k)=1$ for the degree $k$ of the "cyclic cover".

The Calabi-Yau threefolds obtained by $(I)$ are counterexamples to Kodaira vanishing. The surface $X$ required in $(I)$ is precisely as follows:

Corollary 3.4. Let $(X, D)$ be a (pre-)Tango polarized surface with $D=k D^{\prime}$ for some $k \in \mathbb{N}$. Then the Raynaud-Mukai construction gives a polarized Calabi-Yau threefold $(\tilde{X}, \tilde{D})$ by a kth cyclic cover if and only if

(i) $(p, k)=(2,3)$ or $(3,2)$, and

(ii) $D=k D^{\prime}$ for some ample $D^{\prime}$ and $K_{X} \sim D^{\prime}$.

In particular, $X$ is a surface of general type.

Proof. By the same discussion as in the proof of Corollaries 3.2 and 3.3 .

Unfortunately we do not know how to construct a polarized surface $(X, D)$ as in Corollary 3.4. But Theorem 3.5 (i) below seems to indicate a possibility.

Theorem 3.5 (S. Mukai [11]). Let $X$ be a (smooth) surface over the field $k$ of char $k=p>0$. Assume that Kodaira vanishing fails on $X$. Then we have:

(i) $X$ is of general type or a quasi-elliptic surface with Kodaira dimension 1 (if $p=2,3$ ).

(ii) There exists a surface $X^{\prime}$ birationally equivalent with $X$ such that there is a morphism $g: X^{\prime} \longrightarrow C$ to a curve $C$ whose fibers are all connected and singular.

It is proved that, in the case of surfaces, Kodaira (non-)vanishing is preserved in birational equivalence (see Corollary 8 [20]). Thus by Theorem 3.5(ii) it seems to be reasonable to consider a fibration $\rho: X \longrightarrow C$ to a curve.

For a Calabi-Yau threefold, we often assume simple connectedness, which implies that $H^{1}\left(\tilde{X}, \mathcal{O}_{\tilde{X}}\right)=0$ for our example. For this property, we have the following.

Proposition 3.6. Assume that the surface $X$ in Corollary 3.4 has a fibration over a curve $C: g: X \longrightarrow C$ and set $h: \tilde{X} \stackrel{f}{\longrightarrow} X \stackrel{g}{\longrightarrow} C$. Then we have $H^{1}\left(\tilde{X}, \mathcal{O}_{\tilde{X}}\right) \cong H^{1}\left(C, g_{*} \mathcal{O}_{X}\right) \oplus H^{0}\left(C, R^{1} h_{*} \mathcal{O}_{\tilde{X}}\right)$. 
Proof. Consider the Leray spectral sequence

$$
E_{2}^{p q}=H^{p}\left(C, R^{q} h_{*} \mathcal{O}_{\tilde{X}}\right) \Rightarrow H^{p+q}\left(\tilde{X}, \mathcal{O}_{\tilde{X}}\right) .
$$

Then by the 5 -term exact sequence we have

$$
0 \longrightarrow H^{1}\left(C, h_{*} \mathcal{O}_{\tilde{X}}\right) \longrightarrow H^{1}\left(\tilde{X}, \mathcal{O}_{\tilde{X}}\right) . \longrightarrow H^{0}\left(C, R^{1} h_{*} \mathcal{O}_{\tilde{X}}\right) \longrightarrow H^{2}\left(C, h_{*} \mathcal{O}_{\tilde{X}}\right),
$$

where the last term $H^{2}\left(C, h_{*} \mathcal{O}_{\tilde{X}}\right)$ vanishes since $\operatorname{dim} C<2$. Thus we have

$$
H^{1}\left(\tilde{X}, \mathcal{O}_{\tilde{X}}\right) \cong H^{1}\left(C, h_{*} \mathcal{O}_{\tilde{X}}\right) \oplus H^{0}\left(C, R^{1} h_{*} \mathcal{O}_{\tilde{X}}\right) \text {. }
$$

On the other hand, we have $(p, k)=(2,3)$ or $(3,2)$ by Corollary 3.4 and the explicit construction of the cyclic cover gives

$$
\tilde{X}= \begin{cases}\mathcal{S p e c} \bigoplus_{i=0}^{2} \mathcal{O}_{P}(-i) \otimes \pi^{*}\left(2 i D^{\prime}\right) & \text { if }(p, k)=(2,3), \\ \mathcal{S} p e c \bigoplus_{i=0}^{1} \mathcal{O}_{P}(-2 i) \otimes \pi^{*}\left(3 i D^{\prime}\right) & \text { if }(p, k)=(3,2),\end{cases}
$$

where $\pi: P \longrightarrow X$ is the $\mathbb{P}^{1}$-fibering. Thus we compute

$$
\begin{aligned}
h_{*} \mathcal{O}_{\tilde{X}}= & (g \circ \pi \circ \varphi)_{*} \mathcal{O}_{\tilde{X}}=(g \circ \pi)_{*}\left(\varphi_{*} \mathcal{O}_{\tilde{X}}\right) \\
= & \left\{\begin{array}{cl}
(g \circ \pi)_{*}\left(\bigoplus_{i=0}^{2} \mathcal{O}_{P}(-i) \otimes \pi^{*}\left(2 i D^{\prime}\right)\right) & \text { if }(p, k)=(2,3), \\
(g \circ \pi)_{*}\left(\bigoplus_{i=0}^{1} \mathcal{O}_{P}(-2 i) \otimes \pi^{*}\left(3 i D^{\prime}\right)\right) & \text { if }(p, k)=(3,2),
\end{array}\right. \\
= & \left\{\begin{array}{cc}
g_{*}\left(\pi_{*} \mathcal{O}_{P}\right) \oplus g_{*}\left(\pi_{*} \mathcal{O}_{P}(-1) \otimes \mathcal{O}_{X}\left(2 D^{\prime}\right)\right) \\
\oplus g_{*}\left(\pi_{*} \mathcal{O}_{P}(-2) \otimes \mathcal{O}_{X}\left(4 D^{\prime}\right)\right) & \text { if }(p, k)=(2,3), \\
g_{*}\left(\pi_{*} \mathcal{O}_{P}\right) \oplus g_{*}\left(\pi_{*} \mathcal{O}_{P}(-2) \otimes \mathcal{O}_{X}\left(3 D^{\prime}\right)\right) & \text { if }(p, k)=(3,2) .
\end{array}\right.
\end{aligned}
$$

Now since $\pi_{*} \mathcal{O}_{P}=\mathcal{O}_{X}$ and $\pi_{*} \mathcal{O}_{P}(-i)=0$ for $i>0$ we obtain $h_{*} \mathcal{O}_{\tilde{X}}=g_{*} \mathcal{O}_{X}$.

Remark 3.7. Using another spectral sequence and 5-term exact sequence we can show the inclusion $H^{0}\left(C, R^{1} g_{*}\left(f_{*} \mathcal{O}_{\tilde{X}}\right)\right) \subset H^{0}\left(C, R^{1} h_{*} \mathcal{O}_{\tilde{X}}\right)$, but the equality does not hold in general.

Next we consider the construction (II), whose algorithm is as follows: Given a (pre-)Tango curve, we make a (pre-)Tango polarized surface $(X, D)$ and a $\mathbb{P}^{1}$-bundle $\pi: P \longrightarrow X$ with the canonical section $F \subset P$ together with a purely inseparable cover $\left.\pi\right|_{G}: G \longrightarrow X$ of degree $p$ corresponding to $D$. Then choose $k=p^{\ell} r$ with $(p, r)=1$ and $\ell \geq 1$ and let $\varphi: \tilde{X} \longrightarrow P$ be the normalization of $P$ in $k(P)\left(R^{1 / k}\right)$, where $R \in K(P)$ is such that $(R)=G-\left(p F-p \pi^{*}(D)\right)$.

Lemma 3.8. Let $(X, D), D=k D^{\prime}$ with $(2 \leq) k \in \mathbb{N}$, be a (pre-)Tango polarized surface by the Raynaud-Mukai construction. Then the construction (II) gives a Calabi-Yau threefold if and only if $(p, k, K)=\left(2,4,2 D^{\prime}\right)$ or $(3,3, D)$.

Proof. Let $(X, D)$ be a (pre-)Tango polarized surface by the Raynaud-Mukai construction. Then we obtain a $\mathbb{P}^{1}$-bundle $\pi: P \longrightarrow X$ together with the canonical section $F$ and the purely inseparable cover $G \rightarrow X$ of degree $p$ (see Theorem 2.4).

In the Mukai construction, we take a $k$ th cyclic cover of $P$ where $(k, p)=1$. This does not work as we have seen in Corollary 3.3. Thus we assume that $(k, p) \neq 1$ and set $k=p^{\ell} r$ with $(p, r)=1, \ell \geq 1$. Since we have $D=k D^{\prime}$ and $G \sim p F-p \pi^{*}(D)$, there exists $R \in k(P)$ such that $(R)=G-p F+p \pi^{*}\left(k D^{\prime}\right)$. Now let $\varphi: \tilde{X} \longrightarrow P$ be the normalization of $P$ in $k(P)\left(R^{1 / k}\right)$. Then if we set $\tilde{F}=\left(\varphi^{*}(F)\right)_{\text {red }}$ and $\tilde{G}=\left(\varphi^{*}(G)\right)_{\text {red }}$, we have $\varphi^{*}(G)=k \tilde{G}$ and $\varphi^{*}(F)=(k / p) \tilde{F}$ and $\tilde{G} \sim \tilde{F}-p f^{*}\left(D^{\prime}\right)$, 
where $f=\pi \circ \varphi$. Notice that we do not have the coefficient $p$ for $\tilde{F}$ as in the case of $(p, k)=1$. Now as in the proof of Proposition 3.1, we compute

$$
\begin{aligned}
\tilde{K} & \sim \varphi^{*} K_{P}+(k-1) \tilde{G}+\left(\frac{k}{p}-1\right) \tilde{F} \\
& \sim \varphi^{*} K_{P}+\left(k+\frac{k}{p}-2\right) \tilde{F}-p(k-1) f^{*} D^{\prime} \\
& \sim\left(p^{\ell} r-p^{\ell-1} r-2\right) \tilde{F}+f^{*}\left(K+\left(p^{\ell} r-p\left(p^{\ell} r-1\right)\right) D^{\prime}\right) .
\end{aligned}
$$

Then if $\tilde{X}$ is a Calabi-Yau threefold, i.e., $\tilde{K} \sim 0$, we must have $p^{\ell} r-p^{\ell-1} r-2=0$ and $K+\left(p^{\ell} r-p\left(p^{\ell} r-1\right)\right) D^{\prime} \sim 0$, from which we have $(\ell, r, p, k)=(1,1,3,3)$ or $(2,1,2,4)$ and

$$
K \sim \begin{cases}2 D^{\prime} & \text { if }(p, k)=(2,4), \\ 3 D^{\prime}(=D) & \text { if }(p, k)=(3,3) .\end{cases}
$$

Now we can show

Proposition 3.9. Calabi-Yau threefolds cannot be obtained by the construction (II).

Proof. We assume that the (pre-) Tango polarized surface $(X, D)$ is a fibration $f_{1}$ : $X \longrightarrow X_{1}$ over a Tango polarized curve $\left(X_{1}, D_{1}\right)$ with $D_{1}=k_{1} D_{1}^{\prime}$, which is a $k_{1}$ th cyclic cover $\varphi_{1}: X \longrightarrow P_{1}$ of a $\mathbb{P}^{1}$-fibration $\pi_{1}: P_{1} \longrightarrow X_{1}$ ramified over $F_{1}+G_{1} \subset P_{1}$ and we set $\tilde{F}_{1}=\left(\varphi_{1}^{*}\left(F_{1}\right)\right)_{\text {red }}$. In this situation, we have

$$
K \sim\left(p k_{1}-p-k_{1}-1\right) \tilde{F}_{1}+f_{1}^{*}\left(K_{X_{1}}-\left(p k_{1}-p-k_{1}\right) D_{1}^{\prime}\right)
$$

by Proposition 3.1. We have $D=\left(k_{1}-1\right) \tilde{F}_{1}+f_{1}^{*} D_{1}^{\prime}$ by definition. Now we first consider the case $(p, k)=(2,4)$. By Lemma 3.8 we have

$$
2 D^{\prime}=\frac{1}{2} D=\frac{1}{2}\left(k_{1}-1\right) \tilde{F}_{1}+\frac{1}{2} f_{1}^{*} D_{1}^{\prime} \sim K=\left(k_{1}-3\right) \tilde{F}_{1}+f_{1}^{*}\left(K_{X_{1}}-\left(k_{1}-2\right) D_{1}^{\prime}\right)
$$

or otherwise

$$
\frac{5-k_{1}}{2} \tilde{F}_{1}+f_{1}^{*}\left(\frac{2 k_{1}-3}{2} D_{1}^{\prime}-K_{X_{1}}\right) \sim 0
$$

which entails $k_{1}=5$ and $K_{X_{1}}=\frac{7}{2} D_{1}^{\prime}$. But since $D_{1}$ is a (pre-)Tango structure we must have $\frac{7}{2} D_{1}^{\prime}=K_{X_{1}} \geq p D_{1}=2 \cdot 4 D_{1}^{\prime}=8 D_{1}^{\prime}$, a contradiction.

The case of $(p, k)=(3,3)$ is similar. Since we must have $D \sim K$, we have $k_{1}=3$ and $K_{X_{1}}=4 D^{\prime}$. But, since $\left(X_{1}, D_{1}\right)$ is a Tango-Raynaud curve, we must have $4 D_{1}^{\prime}=K_{X_{1}} \geq p D_{1}=3 k_{1} D_{1}^{\prime}=9 D_{1}^{\prime}$, a contradiction.

\section{Cohomology of Calabi-Yau threefold with Tango-structure}

In this section, we compute the cohomology $H^{1}\left(X, H^{-1}\right)$ for an arbitrary ample $H$ under the assumption that $X$ is a Calabi-Yau threefold on which Kodaira vanishing fails.

Theorem 4.1 (N. Shepherd-Barron [17]). Let $X$ be a normal locally complete intersection Fano threefold over the field $k$ of char $k=p \geq 5$ and $L$ be an ample line bundle on $X$. Then we have $H^{1}\left(X, L^{-1}\right)=0$. 
Recall that, for a polarized smooth variety $(X, L)$, Kodaira non-vanishing $H^{1}(X$, $\left.L^{-1}\right) \neq 0$ does not necessarily imply that $L$ is a (pre-)Tango structure. But by Enriques-Severi-Zariski's theorem, there exists $\ell>0$ such that we have $H^{1}(X$, $\left.L^{-p^{\ell+1}}\right)=0$ but $H^{1}\left(X, L^{-p^{\ell}}\right) \neq 0$. Then such an $L^{\ell}$ is at least a pre-Tango structure. Now based on these observations, we obtain

Theorem 4.2. Let $(X, L)$ be a smooth Calabi-Yau threefold over a field $k$ of char $k=p \geq 5$ with Kodaira non-vanishing $H^{1}\left(X, L^{-1}\right) \neq 0$. If $L^{\ell}$ is a Tango structure for some $\ell \geq 1$, then we have

$$
H^{1}\left(X, H^{-1}\right)=H^{0}\left(X, H^{-1} \otimes\left(\rho_{*} \mathcal{O}_{Y} / \mathcal{O}_{X}\right)\right)
$$

for every ample line bundle $H$ on $X$, where $\rho: Y \longrightarrow X$ is a purely inseparable cover of degree $p$ corresponding to the Tango structure as in Theorem 2.4 .

Proof. By taking a sufficiently large power $L^{\ell}, \ell \gg 0$, we can assume from the beginning that $H^{1}\left(X, L^{-p}\right)=0$. Also, by the assumption we can assume that $L$ is a Tango structure. Then by Theorem 2.4 we have a purely inseparable cover $\rho: Y \longrightarrow X$ of degree $p$ and $\omega_{Y} \cong \rho^{*}\left(\omega_{X} \otimes L^{-p+1}\right) \cong\left(\rho^{*} L\right)^{-p+1}$; see II 6.1 .6 [9]. Since $\rho$ is a finite morphism and $L$ is ample, $\rho^{*} L$ is also ample. Thus we know that $Y$ is an integral Fano threefold. Also since $L$ is a Tango structure, $Y$ is smooth by Theorem 2.5. Now let $H$ be an arbitrary ample line bundle on $X$. Then, since $\rho$ is surjective, we have the following exact sequence:

$$
0 \longrightarrow H^{-1} \longrightarrow H^{-1} \otimes \rho_{*} \mathcal{O}_{Y} \longrightarrow H^{-1} \otimes \rho_{*} \mathcal{O}_{Y} / \mathcal{O}_{X} \longrightarrow 0,
$$

from which we obtain the long exact sequence

$$
\begin{aligned}
H^{0}\left(X, \rho_{*} \rho^{*} H^{-1}\right) & \longrightarrow H^{0}\left(X, H^{-1} \otimes \rho_{*} \mathcal{O}_{Y} / \mathcal{O}_{X}\right) \\
& \longrightarrow H^{1}\left(X, H^{-1}\right) \longrightarrow H^{1}\left(X, \rho_{*} \rho^{*} H^{-1}\right) .
\end{aligned}
$$

Now, we have $H^{0}\left(X, \rho_{*} \rho^{*} H^{-1}\right)=H^{0}\left(Y, \rho^{*} H^{-1}\right)=0$ since $\rho$ is finite and $H$ is ample. Also $H^{1}\left(X, \rho_{*} \rho^{*} H^{-1}\right)=H^{1}\left(Y, \rho^{*} H^{-1}\right)$ and this is 0 by Theorem 4.1 .

Recall that for a purely inseparable cover $p: Y \longrightarrow X$ of degree $p$ there exists a $p$-closed rational vector field $D$ on $X$ such that $\left(\rho_{*} \mathcal{O}_{Y}\right)^{D}:=\left\{f \in \rho_{*} \mathcal{O}_{Y}: D(f)=\right.$ $0\}=\mathcal{O}_{X}$ (cf. [15]). Thus we have

Corollary 4.3. Under the same assumption as Theorem 4.2, we have

$$
H^{1}\left(X, H^{-1}\right)=H^{0}\left(X, H^{-1} \otimes D\left(\rho_{*} \mathcal{O}_{Y}\right)\right),
$$

where $D$ is a p-closed rational vector field on $X$ corresponding to the purely inseparable cover $\rho$.

\section{ACKNOWLEDGEMENTS}

The author thanks the referee for many careful comments. He also thanks Prof. Dr. Holger Brenner for stimulating discussions.

\section{REFERENCES}

1. S. Cynk and D. van Straten, Small resolutions and non-liftable Calabi-Yau threefolds. Manuscripta Math. 130 (2009), no. 2, 233-249. MR2545516 (2010k:14074)

2. P. Deligne, Relèvement des surfaces K3 en caractéristique nulle. Prepared for publication by Luc Illusie. Lecture Notes in Math., 868, Algebraic surfaces (Orsay, 1976-78), pp. 58-79, Springer, Berlin-New York, 1981. MR638598 (83j:14034) 
3. P. Deligne and L. Illusie, Relèvements modulo $p^{2}$ et décomposition du complexe de de Rham. Invent. Math. 89 (1987), no. 2, 247-270. MR894379 (88j:14029)

4. T. Ekedahl, On non-liftable Calabi-Yau threefolds (preprint), math.AG/0306435

5. H. Esnault and E. Viehweg, Lectures on vanishing theorems. DMV Seminar, 20. Birkhäuser Verlag, Basel, 1992. MR.1193913 (94a:14017)

6. M. Hirokado, A non-liftable Calabi-Yau threefold in characteristic 3. Tohoku Math. J. (2) 51 (1999), no. 4, 479-487. MR.1725623 (2000m:14044)

7. M. Hirokado, H. Ito and N. Saito, Calabi-Yau threefolds arising from fiber products of rational quasi-elliptic surfaces. I. Ark. Mat. 45 (2007), no. 2, 279-296. MR.2342606 (2008j:14074)

8. M. Hirokado, H. Ito and N. Saito, Calabi-Yau threefolds arising from fiber products of rational quasi-elliptic surfaces. II. Manuscripta Math. 125 (2008), no. 3, 325-343. MR.2373064 (2008m:14078)

9. J. Kollar, Rational Curves on Algebraic Geometry, Ergebnisse der Mathematik und ihre Grenzgebiete 3. Folge, Band 32, Springer, 1996. MR1440180 (98c:14001)

10. M. Maruyama, On a family of algebraic vector bundles, in Number Theory, Algebraic Geometry and Commutative Algebra, in Honor of Y. Akizuki, Kinokuniya, Tokyo, 1973, pp. 95-146. MR 0360587 (50:13035)

11. S. Mukai, On counter-examples of Kodaira's vanishing theorem and Yau's inequality (in Japanese), Kinosaki Algebraic Geometry Symposium, 1979.

12. S. Mukai, Counterexamples of Kodaira's vanishing and Yau's inequality in higher dimensional variety of characteristic $p>0$, RIMS preprint, 2005.

13. D. Mumford, Abelian varieties, Tata Institute of Fundamental Research Studies in Mathematics, 5. Oxford University Press, London, 1970, published for the Tata Institute of Fundamental Research, Bombay. MR0282985 (44:219)

14. M. Raynaud, Contre-exemple au "vanishing theorem" en caractéristique $p>0$ (French). C. P. Ramanujam-a tribute, pp. 273-278, Tata Inst. Fund. Res. Studies in Math., 8, Springer, Berlin-New York, 1978. MR541027 (81b:14011)

15. P. Russell, Factoring the Frobenius morphism of an algebraic surface, Algebraic geometry, Bucharest 1982 (Bucharest, 1982), 366-380, Lecture Notes in Math., 1056, Springer, Berlin, 1984. MR749947 (85m:14055)

16. S. Schröer, Some Calabi-Yau threefolds with obstructed deformations over the Witt vectors. Compos. Math. 140 (2004), no. 6, 1579-1592. MR2098403 (2005i:14051)

17. N. I. Shepherd-Barron, Fano threefolds in positive characteristic. Compositio Math. 105 (1997), no. 3, 237-265. MR.1440723 (98d:14054)

18. Y. Takayama, On non-vanishing of cohomologies of generalized Raynaud polarized surfaces. J. Pure Appl. Algebra 214 (2010), no. 7, 1110-1120. MR2586990 (2011g:14089)

19. Y. Takeda, Pre-Tango structures and uniruled varieties. Colloq. Math. 108 (2007), no. 2, 193-216. MR2291633 (2008g:14026)

20. H. Tango, On the behavior of cohomology classes of vector bundles with regard to Frobenius morphism (Japanese), Suurikaisekikenkyusho Kokyuroku, 1972.

21. H. Tango, On the behavior of extensions of vector bundles under the Frobenius map, Nagoya Math. J., 48 (1972), 73-89. MR0314851 (47:3401)

Department of Mathematical Sciences, Ritsumeikan University, 1-1-1 Nojihigashi, Kusatsu, Shiga 525-8577, Japan

E-mail address: takayama@se.ritsumei.ac.jp 